11. Blumberg N. Deleterious clinical effects of transfusion immunomodulation: proven beyond a reasonable doubt. Transfusion 2005;45(2 Suppl):33S-40S.

12. Silverboard H, Aisiku I, Martin GS, Adams M, et al. The role of acute blood transfusion in the development of acute respiratory distress syndrome in patients with severe trauma. J Trauma 2005;59(3):717-23.

13. Shorr AF, Duh MS, Kelly KM, Kollef MH. Red blood cell transfusion and ventilator-associated pneumonia: a potential link? Crit Care Med 2004;32(3):666-74.
14. Bateman ST, Lacroix J, Boven K, Forbes P, et al. Anemia, blood loss, and blood transfusions in North American children in the intensive care unit. Am J Respir Crit Care Med 2008;178(1):26-33.

15. Valentine SL, Lightdale JR, Tran CM, Jiang H, et al. Assessment of hemoglobin threshold for packed RBC transfusion in a medical-surgical PICU. Pediatr Crit Care Med 2014;15(2):e89-94.

\title{
Publicación de trabajos presentados en los Encuentros Nacionales de Investigación Pediátrica de la Sociedad Argentina de Pediatría: factores involucrados
}

\author{
Publication of abstracts presented at the National Pediatric Research Meetings \\ of the Argentine Society of Pediatrics: Related factors
}

\author{
Dra. Paula Dominguez ${ }^{a}$ Dra. María F. Ossorio ${ }^{a, b}$, Dr. Eduardo Cuestas ${ }^{b, c}$, Dr. Norberto Giglio ${ }^{b, d}$, \\ Dr. Carlos Grandib, , Dr. Facundo García-Bournissen ${ }^{b, d}$, Dr. Santiago Vidaurreta ${ }^{b, f}$, Dr. Jaime Altcheh ${ }^{b, d}$ y \\ Dr. Fernando Ferrero ${ }^{a, b}$
}

\begin{abstract}
RESUMEN
Objetivos: Estimar la proporción de trabajos presentados en Encuentros Nacionales de Investigación Pediátrica que posteriormentefueron publicados en forma completa; describir sus diseños y factores involucrados con la no publicación. Métodos: Estudio descriptivo y analítico, que incluyó todos los trabajos presentados en Encuentros Nacionales de Investigación Pediátrica (1998-2011). Seidentificó un investigador por estudio y se lo encuestó sobre el diseño, la publicación y los factores asociados a la no publicación.

Resultados: Sobre 746 trabajos presentados, respondieron la encuesta los autores de 522 (70\%). El 84,3\% eran estudios observacionales y $15,7 \%$, experimentales; $34 \%$ recibieron financiación. Fueron publicados posteriormente 217 trabajos (41,5\%, IC 95\%: 37,3-45,9). Aquellos con financiación tuvieron
\end{abstract}

a. Hospital General de Niños Pedro de Elizalde, Buenos Aires.

b. Subcomisión de Investigación Pediátrica, Sociedad Argentina de Pediatría.

c. Hospital Privado de Córdoba, Córdoba.

d. Hospital General de Niños Ricardo Gutiérrez, Buenos Aires.

e. Hospital Materno-infantil Ramón Sardá, Buenos Aires.

f. Hospital Universitario CEMIC, Buenos Aires.

Correspondencia: Dra. Paula Domínguez, pau.dom@gmail.com

Financiamiento: La Dra. Paula Domínguez contó con una beca de investigación de la Sociedad Argentina de Pediatría para el desarrollo del estudio.

Conflicto de intereses: Ninguno que declarar.

Recibido: 8-12-2015

Aceptado: 3-2-2016 mayor probabilidad de publicarse (OR 2; IC 95\%: 1,4-2,9; $\mathrm{p}<0,001)$. Las causas de no publicación más frecuentes fueron la falta de tiempo, el tamaño muestral insuficiente y la dificultad con el financiamiento.

Conclusión: El 41,5\% de trabajos presentados en Encuentros Nacionales de Investigación Pediátrica alcanzaron la publicación en texto completo. La falta de tiempo fuela principal causa para no hacerlo.

Palabras clave: bibliografía de medicina, publicaciones periódicas, informe de investigación.

http:/ /dx.doi.org/10.5546/aap.2016.351

\section{INTRODUCCIÓN}

Pocas instituciones se han dedicado más a la salud infantil que la Sociedad Argentina de Pediatría (SAP). Algunas iniciativas pueden mostrar un impacto fácilmente identificable (p.e.: iniciativa sobre retinopatía del prematuro); en otros casos, aun cuando tengan un impacto importante, los resultados no suelen ser tan fáciles de identificar (p.e.: actividades de educación continua). Finalmente, las actividades de investigación promovidas por la SAP, que estimulan el espíritu crítico en los profesionales y generan conocimiento original, permitirán diseñar y/o validar herramientas y estrategias que colaborarán en forma directa con la prevención de la morbimortalidad infantil. En esta iniciativa, se incluyen los Encuentros Nacionales de Investigación Pediátrica que la SAP 
desarrolla desde hace 18 años.

El proceso de investigación solo puede darse por concluido cuando sus resultados son dados a conocer y sometidos a la revisión de pares, lo que hace de la publicación su meta final. Sin embargo, solo una porción limitada de los resúmenes presentados en reuniones científicas alcanza la publicación como artículo completo. ${ }^{1,2}$ Algunas dificultades relacionadas con la publicación han sido descritas, ${ }^{3,}$ ${ }^{4}$ pero su continua identificación puede colaborar en desarrollar estrategias que la faciliten.

En el caso de la SAP, $11 \%$ de los trabajos presentados en su Congreso Nacional alcanza la publicación en forma completa. ${ }^{4}$ Sin embargo, sería razonable pensar que, en eventos de especialidades, se podrían obtener mejores resultados.

Nuestro objetivo fue estimar la proporción de trabajos presentados en Encuentros Nacionales de Investigación Pediátrica de la SAP entre 1998 y 2011 que fueron publicados en forma completa. Además, se describió el diseño de los trabajos y los factores involucrados en la no publicación.

\section{MATERIAL Y MÉTODOS}

Estudio descriptivo y analítico, desarrollado en el segundo semestre de 2014, que incluyó todos los trabajos presentados en Encuentros Nacionales de Investigación Pediátrica (SAP) entre 1998 y 2011. De los libros de resúmenes respectivos, se identificó un investigador por estudio, se lo contactó (por correo electrónico o personalmente) y se le solicitó participar de una encuesta autoadministrada y cerrada, en la que se indagó sobre la publicación del trabajo o los motivos para no hacerlo y las fuentes de financiamiento.
Se consideró publicada una investigación cuando fue efectuada en publicación periódica científica reconocida con ISSN. Además, se verificó si era indizada (Medline o Lilacs) o no.

En aquellos trabajos no publicados, se indagó sobre las posibles causas (falta de interés, falta de tiempo, falta de acuerdo entre coautores, existencia de trabajos con resultados similares u opuestos, impresión de que los resultados no eran trascendentes, tamaño muestral insuficiente, dificultad con el análisis estadístico, pesimismo respecto de su publicación, rechazo, otras) y se solicitó al encuestado que señalara la más importante.

De los resúmenes presentados, se obtuvo el año de presentación, la institución del primer autor, el tipo de investigación (según objetivo: investigación básica, clínica, epidemiológica o de servicios de salud), el diseño (observacional o experimental). Los estudios observacionales se subdividieron en descriptivos y analíticos (transversales, casos y controles, de cohorte), y los experimentales, en ensayos con drogas (fase I, II, III, IV) y sin drogas. También se identificó el alcance (multicéntrico internacional, multicéntrico nacional, local), el estado (presentado como proyecto, trabajo en curso o trabajo finalizado) y la forma de presentación (oral o póster). Los datos de las encuestas fueron vinculados con los de los resúmenes, disociados de datos filiatorios de los investigadores.

Análisis estadístico: Las variables categóricas se expresaron como porcentajes. Utilizamos chi cuadrado para evaluar la asociación entre características de los trabajos (diseño, estado, forma de presentación y financiamiento) y

TABLa 1. Distribución de los trabajos presentados, incluidos en el estudio y publicados, según año

\begin{tabular}{lccccc}
\hline Año & $\begin{array}{c}\text { Presentados } \\
\text { (n) }\end{array}$ & \multicolumn{2}{c}{ Incluidos en el estudio } & \multicolumn{2}{c}{ Publicados } \\
& (n) & (\%) & (n) & (\%) \\
\hline 1998 & 73 & 51 & 69,9 & 22 & 43,1 \\
1999 & 60 & 44 & 73,3 & 16 & 36,4 \\
2001 & 49 & 44 & 89,8 & 22 & 50,0 \\
2002 & 42 & 34 & 81,0 & 18 & 52,9 \\
2003 & 39 & 17 & 43,6 & 6 & 35,3 \\
2004 & 84 & 33 & 39,3 & 12 & 36,4 \\
2005 & 40 & 33 & 82,5 & 10 & 30,3 \\
2006 & 54 & 33 & 61,1 & 21 & 63,6 \\
2007 & 58 & 41 & 70,7 & 19 & 46,3 \\
2008 & 83 & 62 & 74,7 & 20 & 32,3 \\
2009 & 49 & 39 & 79,6 & 17 & 43,6 \\
2010 & 66 & 48 & 72,7 & 19 & 39,6 \\
2011 & 49 & 43 & 87,8 & 15 & 34,9 \\
Total & $\mathbf{4 4 6}$ & $\mathbf{5 2 2}$ & $\mathbf{7 0 , 0}$ & $\mathbf{2 1 7}$ & $\mathbf{4 1 , 6}$ \\
\hline
\end{tabular}


publicación. Calculamos OR con su IC 95\%; nivel de significación de $p<0,05$. Realizamos un análisis multivariado (regresión logística), que incluyó todos los posibles predictores de publicación. Se utilizó SPSS 11.5.

Consideraciones éticas: Se cumplió con la Ley de Protección de Datos Personales ( ${ }^{\circ}$ 25326), que garantiza la adecuada disociación de datos filiatorios. En la encuesta, se informó convenientemente sobre los alcances de la investigación, y se consideró su participación como aceptación. Se obtuvo aprobación de los comités institucionales de la institución a la que pertenecía el primer autor.

\section{RESULTADOS}

En el período estudiado, se presentaron 746 trabajos; respondieron la encuesta los autores de $522(70 \%)$ (Tabla 1$)$.

El 63\% de estos trabajos se encontraban concluidos; $11,7 \%$, en curso; y $25,3 \%$ fueron proyectos de investigación. Se presentaron en forma oral $62,3 \% \mathrm{y}$, como póster, $36,4 \%$. E1 $84,3 \%$ fueron estudios observacionales (43,9\%, descriptivos; $29,5 \%$, transversales; $4 \%$, casos y controles; y $6,9 \%$, de cohorte) y $15,7 \%$, experimentales $(4,5 \%$, ensayos con drogas). Recibieron financiamiento $178(34,1 \%)$ trabajos, en su mayoría (112), de origen público.

Se publicaron en forma completa 217 trabajos (41,5\%, IC 95\%: 37,3-45,9); 83\% lo hizo en revistas indizadas (Lilacs o Medline). Las revistas más mencionadas fueron Archivos Argentinos de
Pediatría (28\%), Medicina (5\%) y Pediatrics (3,6\%).

Las causas más frecuentemente referidas como responsables de la no publicación fueron la falta de tiempo ( $\mathrm{n}=91 ; 29,8 \%)$, el tamaño muestral insuficiente $(\mathrm{n}=34 ; 11,1 \%)$, la dificultad con el financiamiento $(n=27 ; 8,8 \%)$, el pesimismo de los autores con respecto a su publicación $(\mathrm{n}=20 ; 6,5 \%)$ y considerar los resultados como no trascendentes $(n=19 ; 6,2 \%)$. Ninguno refirió haber sido rechazado como causa para la no publicación.

Tuvieron mayor probabilidad de publicarse los trabajos con financiamiento y que se encontraban concluidos. No se encontró asociación entre el diseño experimental ni la modalidad de presentación oral con ulterior publicación (Tabla 2).

Luego de controlar por potenciales predictores, recibir financiamiento y estar concluido al momento de la presentación continuaron siendo predictores independientes de publicación (Tabla 3).

\section{DISCUSIÓN}

Encontramos que $41,5 \%$ de los trabajos presentados en Encuentros Nacionales de Investigación Pediátrica (SAP) fueron publicados luego como texto completo. Este dato se acerca a la tasa promedio global $(44,5 \%),{ }^{2}$ pero fue superior al referido para Congresos Argentinos de Pediatría (CONARPE) (11,3\%). ${ }^{4}$ Probablemente, esta diferencia esté relacionada con el tipo de trabajos aceptados en los CONARPE, en los que es frecuente la presentación de casos clínicos, modelo que tiene menor posibilidad

Tabla 2. Análisis de potenciales predictores de publicación de los trabajos analizados

\begin{tabular}{llccccc}
\hline & & Publicados & No publicados & OR & IC 95\% & $p^{*}$ \\
\hline Estado & Concluido & $175(33,5)$ & $215(41,2)$ & 1,7 & $1,1-2,6$ & $<0,01$ \\
& Proyecto & $42(8,0)$ & $90(17,2)$ & & & \\
Presentación & Oral & $142(27,2)$ & $183(35,1)$ & 1,2 & $0,8-1,8$ & 0,2 \\
& Póster & $75(14,4)$ & $122(23,4)$ & & & \\
Diseño & Experimental & $40(7,7)$ & $42(8,0)$ & 1,4 & $0,8-2,7$ & 0,1 \\
& Observacional & $177(33,9)$ & $263(50,4)$ & & & \\
Financiamiento & Sí & $94(18,0)$ & $84(16,1)$ & 2 & $1,1-2,9$ & $<0,001$ \\
& No & $123(23,6)$ & $221(42,3)$ & & & \\
\hline
\end{tabular}

* Chi cuadrado. OR: odds ratio; IC: intervalo de confianza.

TABLA 3. Análisis multivariado de potenciales predictores de publicación

\begin{tabular}{lccc}
\hline Variable & OR & IC 95\% & $p$ \\
\hline Trabajo concluido & 1,97 & $1,27-3,07$ & 0,02 \\
Presentación oral & 1,44 & $0,97-2,14$ & 0,06 \\
Diseño experimental & 1,35 & $0,82-2,23$ & 0,23 \\
Financiamiento & 2,01 & $1,38-2,92$ & $<0,01$ \\
\hline
\end{tabular}

OR: odds ratio; IC: intervalo de confianza. 
de alcanzar la publicación y que no es aceptado en los Encuentros Nacionales de Investigación Pediátrica, y con el diferente perfil de los participantes en ambos eventos.

Nuestro resultado fue similar al encontrado en los congresos de distintas especialidades médicas. Al analizar otras sociedades de especialidades pediátricas de Europa y Estados Unidos, se evidencia que la tasa de publicación de los trabajos presentados en sus respectivas reuniones anuales varía entre $36 \%$ y $60 \%{ }^{5,6,7}$ Es de esperar que los congresos más especializados se asocien con mayor tasa de publicación, como, en nuestro medio, las Reuniones de la Sociedad Latinoamericana de Investigación Pediátrica, en las que alcanza $58 \%{ }^{8}$

La revista más elegida para publicar los estudios fue Archivos Argentinos de Pediatría. Creemos que esto puede estar relacionado con la amplia difusión de esta publicación en nuestro medio, aunque la facilidad de utilizar el propio idioma también podría haber influido. ${ }^{9}$

Contar con financiamiento estuvo significativamente asociado a la publicación, y no influyeron ni el diseño ni el tipo de presentación, aunque, en este último caso (presentación oral), la ausencia de significación puede deberse a una falta de potencia $(p=0,06)$. Kleine-Konig ${ }^{6}$ y Shamliyan ${ }^{10}$ encontraron que los trabajos experimentales y aquellos que se presentaban en forma oral se publicaban más que los observacionales. También Canosa ${ }^{4}$ encontró que la presentación oral se asociaba a mayor posibilidad de publicación. Es posible que las características de los trabajos presentados en nuestros eventos, exclusivamente trabajos de investigación, hayan hecho que la financiación tuviera más influencia en la posibilidad de alcanzar la publicación. ${ }^{8}$

Coincidiendo con otros estudios, la falta de tiempo fue el factor más referido por los autores como causa de la no publicación, ${ }^{11}$ lo que remarca la necesidad de considerar el tiempo para alcanzar la publicación en el cronograma de cada proyecto de investigación y la importancia de contar con tiempo protegido para la investigación dentro de la práctica profesional. ${ }^{12} \mathrm{De}$ igual forma, se debe destacar la importancia de un buen análisis de factibilidad previo, que contemple lo necesario para alcanzar el tamaño muestral requerido.

Nuestro estudio podría incluir las potenciales debilidades de este tipo de investigaciones. Si bien no logramos encuestar a la totalidad de los autores, tuvimos una elevada tasa de respuesta $(70 \%)$. Todos los datos referidos a las publicaciones fueron verificados en las revistas correspondientes. Además, se incluyeron presentaciones realizadas hasta tres años antes, teniendo en cuenta que la mayoría de los trabajos se publican dentro de los 2 años posteriores a su presentación. ${ }^{13}$

Nuestros resultados brindan información que colaborará a la hora de diseñar estrategias destinadas a incrementar la tasa de publicación.

\section{CONCLUSIÓN}

Más del $40 \%$ de trabajos presentados en Encuentros Nacionales de Investigación alcanzaron la publicación en texto completo. Recibir financiamiento y estar concluidos al momento de la presentación incrementaron la posibilidad de publicación. La falta de tiempo fue el factor más referido como impedimento para alcanzarla.

\section{REFERENCIAS}

1. Von Elm E, Costanza MC, Walder B, Tramer MR. More insight into the fate of biomedical meeting abstracts: a systematic review. BMC Med Res Methodol 2003;3:12.

2. Scherer RW, Langenberg P, von Elm E. Full publication of results initially presented in abstracts. Cochrane Database Syst Rev 2007;(2):MR000005.

3. Harling L, Craig WR, Russell K, Stevens K,et al. Factors influencing the publication of randomized controlled trials in child health research. Arch Pediatr Adolesc Med 2004;158(10):983-7

4. Canosa D, Ferrero F, Melamud A, Otero P, et al. Publicación completa de trabajos presentados en el $33^{\circ}$ Congreso Argentino de Pediatria y análisis de factores queimpidieron su publicación. Arch Argent Pediatr 2011;109(1):56-9.

5. Macdonald AL, Parsons C, Davenport M. Outcome of abstracts presented at the British Association of Paediatric Surgeons congresses (1999-2008). J Pediatr Surg 2012;47(2):386-90.

6. Kleine-Konig MT, Schulte TL, Gosheger G, Rödl R, et al. Publication rate of abstracts presented at European Paediatric Orthopaedic Society Annual Meetings, 2006 to 2008. J Pediatr Orthop 2014;34(6):e33-8.

7. Salami K, Alkayed K. Publication bias in pediatric hematology and oncology: analysis of abstracts presented at the annual meeting of the American Society of Pediatric Hematology and Oncology. Pediatr Hematol Oncol 2013;30(3):165-9.

8. Dicembrino M, Anderson M, Vely AG, Ossorio MF, et al. Publicación completa de trabajos presentados en reuniones de una sociedad científica latinoamericana. Arch Argent Pediatr 2014;112(6):532-6.

9. Otero P. El proceso de inclusión de Archivos en la base de datos Medline. Arch Argent Pediatr 2009;107(1):2-3.

10. Shamliyan T,Kane RL. Clinical research involving children: registration, completeness and publication. Pediatrics 2012;129(5):e1291-300.

11. Scherer RW, Ugarte-Gil C, Schmucker C, Meerpohl JJ. Authors report lack of time as main reason for unpublished research presented at biomedical conferences: a systematic review. J Clin Epidemiol 2015;68(7):803-10.

12. Dang Do AN, Munchhof AM, Terry C, Emmett T, et al. Research and publication trends in hospital medicine. $J$ Hosp Med 2014;9(3):148-54.

13. Gregory TN, Liu T, Machuk A, Arneja JS. What is the ultimate fate of presented abstracts? The conversion rates of presentations to publications over a five-year period from three North American plastic surgery meetings. Can J Plast Surg 2012;20(1):33-6. 